\title{
Real-World Effectiveness of Ivabradine in Chinese Patients with Chronic Heart Failure: Interim Analysis of the POSITIVE Study
}

\author{
Zhou Jingmin $^{1} \cdot$ Xu Yamei $^{1} \cdot$ Zhang Yuhui ${ }^{2} \cdot$ Ding Wenhui ${ }^{3} \cdot$ Tang Baopeng $^{4} \cdot$ Qian Caizhen $^{5} \cdot$ Han Huiyuan ${ }^{6}$. \\ Ge Junbo ${ }^{1}$ on behalf of The POSITIVE investigators
}

Accepted: 3 September 2021 / Published online: 8 December 2021

(c) The Author(s) 2021

\begin{abstract}
Background Ivabradine improves cardiac function and clinical outcomes in chronic heart failure (HF) by reducing heart rate (HR), but there is a lack of real-world data on its effectiveness and safety in Chinese patients.

Methods We designed a prospective, multicenter, observational study of Chinese adults with HF and left ventricular systolic dysfunction, resting HR $\geq 75$ beats per minute (bpm), and an indication for ivabradine treatment. An interim analysis was performed using a cut-off date of 31 October 2019. The primary outcome was change in HR at 6 months after the initiation of ivabradine. Secondary endpoints included change in New York Heart Association (NYHA) functional class; quality of life (QoL), measured using the Kansas City Cardiomyopathy Questionnaire (KCCQ); and adverse events (AEs).

Results Overall, 655 subjects were included in the interim analysis. Mean reduction in HR from baseline was $13.2(95 \%$ confidence interval [CI] 11.2-15.2) bpm at Month 1, and 14.5 (95\% CI 11.8-17.2) bpm at Month 6 ( $p<0.001$ for both changes). NYHA functional class and KCCQ scores improved significantly over time $(p<0.001$ for all comparisons with baseline), indicating amelioration of symptoms and better QoL, respectively. Forty-four subjects (6.7\%) reported a total of 60 ivabradine-related AEs, most frequently phosphenes and bradycardia (both $n=6,0.9 \%$ ).

Conclusion Treatment with ivabradine for 6 months effectively reduced HR and improved functional class and QoL in Chinese patients with chronic HF. Treatment was well tolerated.

Clinical Trial Registration ISRCTN11703380; registered on 8 November 2016.
\end{abstract}

The POSITIVE investigators are mentioned in the Appendix section

\section{Ge Junbo}

jbge@zs-hospital.sh.cn

1 Shanghai Institute of Cardiovascular Diseases, Zhongshan Hospital, Fudan University, \#180 Fenglin Road, Xuhui District, Shanghai 200032, China

2 Fuwai Hospital, Chinese Academy of Medical Sciences and Peking Union Medical College, Beijing, China

3 Peking University First Hospital, Beijing, China

4 The First Affiliated Hospital of Xinjiang Medical University, Ürümqi, China

5 Zhuji People's Hospital of Zhejiang Province, Zhuji Affiliated Hospital of Wenzhou Medical University, Zhejiang, Zhuji, China

6 Shanxi Cardiovascular Hospital, Shanxi, Taiyuan, China

\section{Key Points}

We undertook an interim analysis of an observational study in 655 Chinese patients who were prescribed ivabradine for the treatment of chronic heart failure.

Over 6 months, ivabradine was associated with significant reductions in heart rate and significant improvements in New York Heart Association functional class and quality of life.

Ivabradine was very well tolerated, with $<10 \%$ of patients experiencing treatment-related adverse events. 


\section{Introduction}

In patients with chronic heart failure (HF), compensatory increases in heart rate (HR) not only maintain adequate cardiac output but also increase the risk of disease progression. Persistent elevations in HR can lead to decreased myocardial contractility [1], increased ventricular afterload [2], reduced myocardial perfusion [3], and increased myocardial oxygen consumption [4]. These factors lead to a progressive decline in cardiac function, recurrent dyspnea and peripheral edema, increased levels of B-type natriuretic peptide (BNP), and myocardial remodeling [5]. In turn, worsening HF leads to further increases in HR via activation of the neurohumoral system, creating a self-propagating cycle of events that increases the risk of hospitalization and death.

Data from observational studies $[6,7]$ and SHIFT (Systolic Heart failure treatment with the $\mathrm{I}_{f}$ inhibitor ivabradine Trial) [8] have demonstrated that increased HR is a predictor of short- and long-term mortality in patients with HF. Consequently, HR has become an established therapeutic target in guidelines on HF treatment [9-12]. In patients with HF with reduced ejection fraction (HFrEF), reductions in HR lead to improvements in cardiac function; this in turn leads to a reduction in symptoms and improved outcomes $[13,14]$.

Ivabradine is a novel treatment for HF that reduces HR by selectively inhibiting the $I_{\mathrm{f}}$ current in the sinoatrial node [10]. In 2010, the SHIFT study demonstrated that ivabradine, added to standard therapy, significantly reduced the risk of hospitalization due to worsening $\mathrm{HF}$ in patients with HFrEF, compared with placebo [14]. Subsequently, ivabradine treatment was incorporated into clinical guidelines for treatment intensification in patients who, despite guideline-directed HF treatment including an optimized $\beta$-blocker, have symptomatic HFrEF (New York Heart Association [NYHA] class II or III, with left ventricular ejection fraction $[\mathrm{LVEF}] \leq 35 \%$ ) and a resting HR of $\geq 70$ beats per minute (bpm) in sinus rhythm [11].

Observational studies of ivabradine conducted in Germany (INTENSIFY [15] and RELIf-CHF [16]) and the UK (LIVE:LIFE [17]) have confirmed that ivabradine improves symptoms and health-related quality of life (QoL) in patients with HF in real-world clinical practice. However, to date, there is little published real-world evidence to support the use of ivabradine in Chinese patients with HF. We therefore designed an observational study to assess the effectiveness and safety of ivabradine in this population.

The POSITIVE (POSt-authorization drug Intensive surveillance monitoring sTudy of IVabradinE in patients with HF) study is the first major observational study of ivabradine to be undertaken in China, and indeed is the first large study of ivabradine to assess real-world outcomes of HF in China. Its purpose is to assess the effectiveness and safety of ivabradine in Chinese patients with HFrEF. In this article, we present the findings of an interim analysis of the POSITIVE database.

\section{Methods}

The POSITIVE study is an ongoing, prospective, observational study of patients with HFrEF receiving care at one of 63 hospitals in China (see Appendix for full list). To be included, patients needed to have an LVEF $\leq 40 \%$ in the previous 3 months; NYHA class II, III or IV HF; and a resting HR $\geq 75 \mathrm{bpm}$ in sinus rhythm. In addition, patients had to be receiving standard treatment for HF ( $\beta$-blockers, or other agents if $\beta$-blockers were not tolerated or were contraindicated); meet the indication criteria for ivabradine in China; and to have been prescribed ivabradine by their treating physician.

According to prescribing guidelines in China, the starting dose of ivabradine is $5 \mathrm{mg}$ twice daily, or $2.5 \mathrm{mg}$ twice daily in patients aged $>75$ years. The dosage can subsequently be increased if necessary, based on HR measurement, to a maximum of $7.5 \mathrm{mg}$ twice daily. In the current study, investigators could adjust the dosage based on the patient's condition and according to the dosage recommendations in the prescribing information. Patients with a resting HR of 50-60 bpm continued to receive the starting dose, whereas patients with a resting HR that was persistently $>60 \mathrm{bpm}$ could have the ivabradine dose increased up to a maximum of $7.5 \mathrm{mg}$ twice daily. The ivabradine dose was decreased or treatment discontinued if the patient's resting HR was persistently < $50 \mathrm{bpm}$ or if they had symptoms of bradycardia.

As per usual clinical practice for the monitoring of patients receiving care for HFrEF, patients had monthly follow-up visits, during which the ivabradine dosage could be adjusted. In this observational study, data on clinical status were collected at two follow-up visits, at 1 month (M1) and 6 months (M6) after inclusion. If patients were unable to attend the hospital for follow-up, they were interviewed by telephone. At baseline and at each follow-up visit, patients underwent a physical examination and blood was taken for routine laboratory analysis. NYHA class was assessed and the validated Chinese-language version of Kansas City Cardiomyopathy Questionnaire (KCCQ) [18] was administered to monitor changes in health-related QoL over time. In the KCCQ, 23 items are rated and scaled to generate a score of between 0 and 100, where lower scores indicate poor QoL and high scores indicate good QoL. The minimum change in KCCQ score that is considered clinically meaningful is 5 points [19]. Patients were also requested to keep a diary of 
any adverse events (AEs) and were asked about these AEs during telephone follow-up. Bradycardia that was symptomatic or characterized by an HR of $<40 \mathrm{bpm}$ was recorded as an $\mathrm{AE}$.

The primary outcome of interest was the change in HR between baseline and each of the follow-up visits. Secondary outcomes were the changes in NYHA functional class and KCCQ scores, as well as safety and tolerability. Changes in blood BNP/N-terminal pro-BNP (NT-proBNP) levels and LVEF were also assessed.

Informed consent to participate in this study was obtained from all participants. The study was approved by the Ethics Committee of Zhongshan Hospital Fudan University, and also underwent ethical review and approval at all participating centers. The trial was registered on the ISRCTN database (registration number: 11703380) on November 8, 2016.

An interim analysis was planned for when $\geq 500$ patients had been enrolled and had completed two follow-up visits. In practice, we applied a cut-off date of October 31, 2019, and analyzed data for all patients enrolled up to that point.

Statistical analysis was performed using SAS version 9.1.3 (SAS Institute, Inc., Cary, NC, USA). The full analysis set (FAS) included all enrolled patients who provided informed consent and received at least one dose of ivabradine. The efficacy population consisted of the FAS population who took ivabradine for at least 1 month (i.e., excluding those who discontinued or changed ivabradine at the M1 visit or prematurely discontinued prior to the M1 visit). The primary efficacy analysis was carried out in the efficacy population. Unless otherwise specified, analysis of patient demographics, secondary efficacy endpoints and safety endpoints were carried out in the FAS. For continuous variables, either the mean and standard deviation (SD) or the median and interquartile range (IQR) were calculated. Categorical variables were summarized using frequency, which was expressed as an absolute number $(n)$ and percentage (\%). To analyze differences in outcomes of interest between followup visits and baseline, we used either the paired Student's $t$-test or the Wilcoxon signed-rank test, depending on the distribution of the data; for ranked variables (such as NYHA class), we used the Wilcoxon signed-rank test. A $p$-value $<0.05$ was considered to be statistically significant.

\section{Results}

\subsection{Baseline Characteristics}

At October 31, 2019, 655 patients had been enrolled in the study and were included in the FAS interim analysis. Of these, $554(84.6 \%)$ and $449(68.5 \%)$ subjects had completed the M1 and M6 follow-up visits, respectively. The efficacy analysis included 534 patients; within this group, baseline ECG HR data were missing for 23 patients, and baseline KCCQ data were missing for 37 patients. Baseline NYHA class data were available for all 534 patients in the efficacy population. Data were missing at M1 and M6 for 306 and 393 patients, respectively, for HR; 122 and 227 patients, respectively, for NYHA class; and 156 and 253 patients, respectively, for KCCQ score.

Baseline demographic and clinical characteristics of the study population are shown in Table 1. Participants had a mean $( \pm$ SD) age of $54.9( \pm 15.0)$ years, and most were male $(n=507,77.4 \%)$. The median time since HF diagnosis was 5 months and the mean $( \pm \mathrm{SD})$ LVEF was $30.9( \pm 7.2) \%$. Most (> 75\%) patients had a baseline HR of $80 \mathrm{bpm}$ or higher, but mean $( \pm \mathrm{SD})$ systolic and diastolic blood pressures were normal $(116.3[ \pm 17.3] \mathrm{mmHg}$ and 74.9 [ \pm 12.3$] \mathrm{mmHg}$, respectively). In $81.8 \%$ of cases $(n=536)$, HF was due to either dilated cardiomyopathy or coronary heart disease; collectively, hypertension, valvular heart disease, and myocarditis accounted for a minority $(n=81,12.3 \%)$ of cases. Most patients had either NYHA class II or III HF $(n=511,78.0 \%)$ and almost all were receiving treatment with a $\beta$-blocker $(85.3 \%)$ and/or an aldosterone receptor antagonist $(88.5 \%)$.

At baseline, most patients were receiving ivabradine at a dose of $2.5 \mathrm{mg}(n=363,55.4 \%)$ or $5 \mathrm{mg}(n=288$, $44.0 \%$ ), and most patients received twice-daily administration $(n=620,94.7 \%)$. Similarly, at M1, most patients received a dose of $2.5 \mathrm{mg}(n=211 / 509,41.5 \%)$ or 5 $\mathrm{mg}(n=262 / 509,51.5 \%)$, administered twice daily $(n=488 / 509,95.9 \%)$. Changes in study drug administration from the previous visit were as follows: 369 patients (68.8\%) had no change in dose, 120 (22.4\%) had a dose increase, 21 (3.9\%) had a dose decrease, and 26 (4.9\%) discontinued treatment. At M6, $2.5 \mathrm{mg}(n=131 / 354$, $37.0 \%)$ and $5 \mathrm{mg}(n=192 / 354,54.2 \%)$ continued to be the most commonly used doses, and most patients received twice-daily administration $(n=335 / 354,94.6 \%)$. At this time point, changes in study drug administration from the previous visit were: 309 patients $(73.0 \%)$ had no change of dose, 25 (5.9\%) had a dose increase, 19 (4.5\%) had a dose decrease, and $70(16.5 \%)$ discontinued treatment.

\subsection{Heart Rate}

Mean HR $( \pm$ SD) declined from $88.9( \pm 12.4)$ bpm at baseline to $75.0( \pm 12.7) \mathrm{bpm}$ at M1 and $73.8( \pm 12.8)$ $\mathrm{bpm}$ at M6, representing decreases of 13.2 (95\% confidence interval [CI] 11.2-15.2) bpm between baseline and M1, and 14.5 (95\% CI 11.8-17.2) bpm between baseline and M6 (Fig. 1a). The reductions in HR at both follow-up visits were statistically significant $(p<0.001)$. 
Table 1 Demographics and baseline clinical data of the 655 patients included in the full analysis set of the interim analysis of the POSITIVE study

\begin{tabular}{|c|c|}
\hline Variable & Value \\
\hline Age, years & $54.9(15.0)$ \\
\hline \multicolumn{2}{|l|}{$\operatorname{Sex}[n(\%)]$} \\
\hline Male & $507(77.4)$ \\
\hline Female & $148(22.6)$ \\
\hline Duration of heart failure, months ${ }^{\mathrm{a}}$ & $5.0(2.0-25.0)$ \\
\hline \multicolumn{2}{|l|}{ Cause of heart failure $[n(\%)]$} \\
\hline Dilated cardiomyopathy & 299 (45.6) \\
\hline Coronary heart disease & $237(36.2)$ \\
\hline Hypertension & $52(7.9)$ \\
\hline Valvular heart disease & $21(3.2)$ \\
\hline Myocarditis & $8(1.2)$ \\
\hline Other & $38(5.8)$ \\
\hline Heart rate, bpm & $88.9(12.4)$ \\
\hline \multicolumn{2}{|l|}{ Heart rate category, bpm $[n(\%)]^{\mathrm{b}}$} \\
\hline 75 to $<80$ & $147(22.4)$ \\
\hline 80 to $<90$ & $226(34.5)$ \\
\hline 90 to $<100$ & $139(21.2)$ \\
\hline$\geq 100$ & $106(16.2)$ \\
\hline \multicolumn{2}{|l|}{ Blood pressure, $\mathrm{mmHg}$} \\
\hline Systolic & $116.3(17.3)$ \\
\hline Diastolic & $74.9(12.3)$ \\
\hline \multicolumn{2}{|l|}{ Symptoms of heart failure $[n(\%)]$} \\
\hline Dyspnea & $544(83.1)$ \\
\hline At rest & $82(12.5)$ \\
\hline Exertional & $404(61.7)$ \\
\hline Paroxysmal nocturnal dyspnea & $171(26.1)$ \\
\hline Other dyspnea & $11(1.7)$ \\
\hline Fatigue & $516(78.8)$ \\
\hline \multicolumn{2}{|l|}{ NYHA functional class $[n(\%)]$} \\
\hline $\mathrm{I}^{\mathrm{c}}$ & $2(0.3)$ \\
\hline II & $199(30.4)$ \\
\hline III & $312(47.6)$ \\
\hline IV & $142(21.7)$ \\
\hline Left ventricular ejection fraction, $\%$ & $30.9(7.2)$ \\
\hline \multicolumn{2}{|l|}{ Concomitant treatments $[n(\%)]$} \\
\hline ACE inhibitor & $257(39.2)$ \\
\hline Angiotensin II receptor blocker & $171(26.1)$ \\
\hline$\beta$-blocker & $559(85.3)$ \\
\hline Aldosterone receptor antagonist & $580(88.5)$ \\
\hline Loop diuretics & $544(83.1)$ \\
\hline Hydrochlorothiazide & $40(6.1)$ \\
\hline Digoxin & $188(28.7)$ \\
\hline Device therapy $[n(\%)]$ & $16(2.4)$ \\
\hline Implanted cardioverter defibrillator & $9(1.4)$ \\
\hline Pacemaker & $7(1.1)$ \\
\hline
\end{tabular}

Continuous variables are expressed as mean \pm standard deviation unless otherwise stated

$A C E$ angiotensin-converting enzyme, bpm beats per minute, $N Y H A$ New York Heart Association
Table 1 (continued)

${ }^{a}$ Data are expressed as median (interquartile range)

${ }^{\mathrm{b}}$ At baseline, 37 subjects could not be grouped according to heart rate because of a lack of ECG data

${ }^{\mathrm{c}}$ According to the enrollment criteria, patients with NYHA functional class I should not have been included in the study; however, two such patients (both inpatients) were enrolled. Analysis of NYHA cardiac function is based on the full analysis set.

\subsection{Symptoms and Cardiac Function}

NYHA class showed significant improvements at both follow-up visits compared with baseline, with all $p$-values $<0.001$ (Fig. 1b). Over the course of 6 months, the proportion of patients with NYHA class III or IV HF decreased, whereas the proportion of patients with NYHA class I or II HF increased. An improvement in NYHA class of at least one class relative to baseline was seen in $58.3 \%$ of subjects at M1 and $63.1 \%$ of subjects at M6.

Levels of BNP and NT-proBNP decreased during the study (Fig. 2), but the reductions from baseline were not statistically significant. LVEF tended to increase: median (IQR) LVEF was 31.0\% (26.0-36.0\%) at baseline, 35.0\% (29.0-43.0\%) at M1, and $41.0 \%(35.0-52.9 \%)$ at M6.

\subsection{Quality of Life}

Mean KCCQ clinical symptom and total scores of KCCQ were significantly higher at both M1 and M6 compared with baseline ( $p<0.001$ for all comparisons). Most patients had changes of $\geq 5$ points in clinical symptom and total scores between baseline and both follow-up visits $(65.5 \%$ at $\mathrm{M} 1$ and $71.4 \%$ at $\mathrm{M} 6$, and $65.3 \%$ at $\mathrm{M} 1$ and $73.9 \%$ at M6, respectively), indicating clinically meaningful improvements in QoL.

\subsection{Safety and Tolerability}

In total, 359 AEs occurred in 205 subjects (31.3\%) (Table 2). All AEs were treatment-emergent, and 60 (reported by 44 subjects [6.7\%]) were considered to be related to ivabradine. The most common drug-related AEs were phosphenes and bradycardia, which occurred in six patients each $(0.9 \%)$. Eleven patients developed a total of 14 serious drug-related AEs, including atrial fibrillation $(n=3,0.5 \%)$, HF $(n=2,0.3 \%)$, hypotension $(n=2$, $0.3 \%)$, sinus bradycardia $(n=1,0.2 \%)$, ventricular extrasystole $(n=1,0.2 \%)$, bradycardia $(n=1,0.2 \%)$, myocardial infarction $(n=1,0.2 \%)$, palpitations $(n=1,0.2 \%)$, prolonged QT interval on ECG $(n=1,0.2 \%)$, and atopic dermatitis $(n=1,0.2 \%)$. 


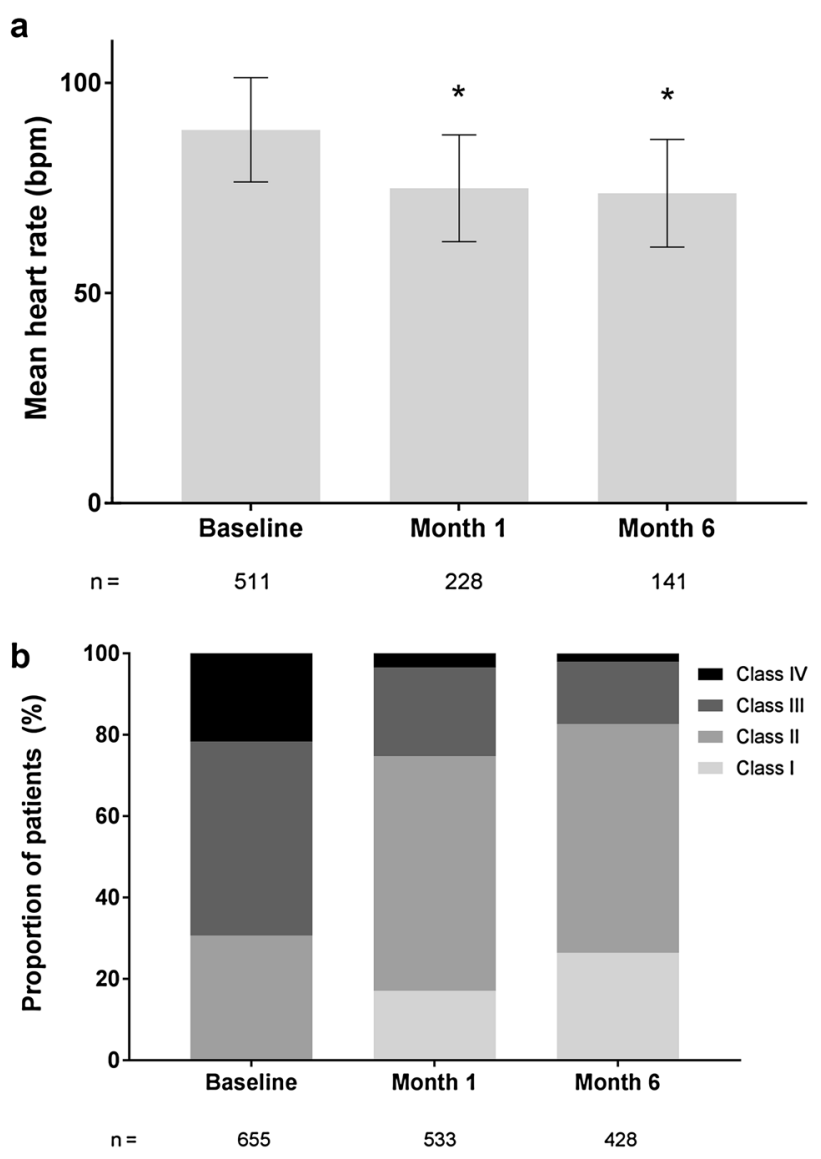

C

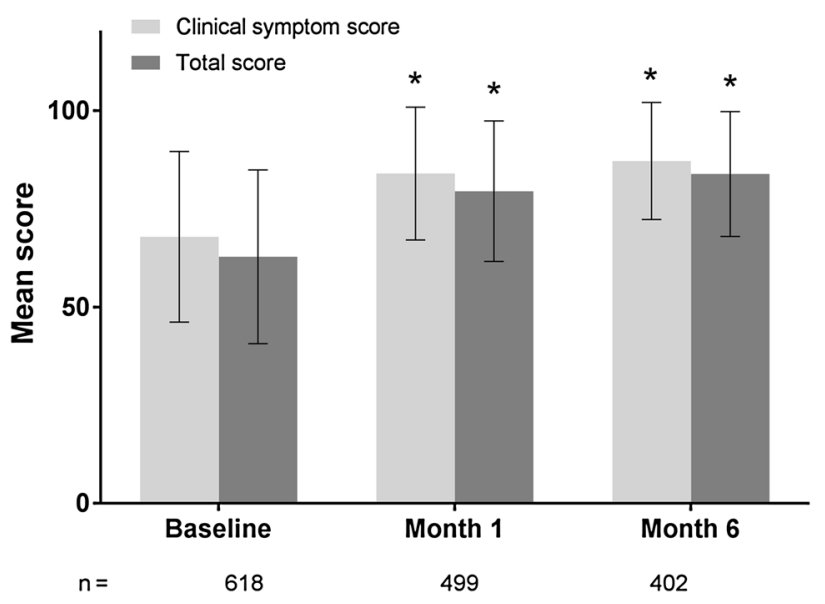

Fig. 1 a Mean heart rate; b New York Heart Association (NYHA) functional class; and c mean Kansas City Cardiomyopathy Questionnaire scores at baseline and at the two follow-up visits (Month 1 and Month 6). Whiskers in (a, c) signify standard deviation. $* p<0.001$ versus baseline. $b p m$ beats per minute

\section{Discussion}

We report interim data from the first large real-world study of ivabradine in Chinese patients with HFrEF. In our cohort of 655 patients, ivabradine significantly reduced mean HR by 13.2 (95\% CI $11.2-15.2) \mathrm{bpm}$ and 14.5 (95\% CI 11. 8-17.2) bpm after 1 and 6 months of treatment, respectively. In comparison, placebo-corrected reductions in HR among ivabradine recipients in the SHIFT trial were 10.9 (95\% CI $10.4-11.4) \mathrm{bpm}$ for the overall study population $(n=3241)$ [13], and 12.1 (95\% CI 8.9-15.3) bpm among the Chinese subpopulation $(n=225)$ [20].

We also found clinically important improvements in NYHA class over 6 months, with approximately $60 \%$ of patients experiencing an improvement of at least one NYHA class (e.g., class III to class II) at both follow-up visits. Concomitant reductions in HR and improvements in NYHA class were also found in the SHIFT trial [8] of ivabradine and in the real-world INTENSIFY study [15].

Treatment with ivabradine also improved QoL in our study, as indicated by increases in KCCQ scores over time. This finding is consistent with the results of previous prospective [15-17] and retrospective [21] real-world studies of ivabradine in patients with HF. These findings are important not only because improving symptoms and QoL is a major goal of HF management [12, 22], but also because such improvements can have prognostic significance [23-26]. For example, in the SHIFT trial [26], a low baseline KCCQ score was predictive of cardiovascular death or hospital admission in patients with NYHA class II-IV systolic HF [26].

We found trends towards reductions in blood BNP and NT-proBNP levels, and towards improvements in LVEF. Because of the observational nature of our study, monitoring reflected routine clinical practice in China, and the fact that current national guidelines on the management of HF do not recommend regular echocardiography or routine measurement of BNP or NT-proBNP levels [22]. Thus, many of our patients had missing values and this may explain why the trends we observed did not reach statistical significance.

Treatment-emergent AEs were broadly consistent with the known tolerability profile of ivabradine and there were no new or unexpected findings in relation to drug safety. Our findings therefore suggest that ivabradine is generally well tolerated in Chinese patients with HFrEF.

Ivabradine is an important addition to the available treatment options for HF. $\beta$-blockers, although effective in reducing HR, alter myocardial contractility and intracardiac conduction; this can preclude their use or prevent dose optimization in some patients. In contrast, ivabradine reduces HR without affecting conduction or contractility [8]. In addition to the clinical effects described above, ivabradine has been found to improve left ventricular remodeling [14], increase exercise tolerance [27], and reduce the risk of HFrelated death [13].

The mechanism by which HR reduction improves outcomes in HF has been investigated. A key finding has been that, in HF, myocardial contractility is inversely 
Fig. 2 Median plasma levels of BNP and NT-proBNP at baseline, Month 1 and Month 6. Whiskers signify the interquartile range. $B N P$ B-type natriuretic peptide, $N T$-proBNP $\mathrm{N}$-terminal pro-BNP

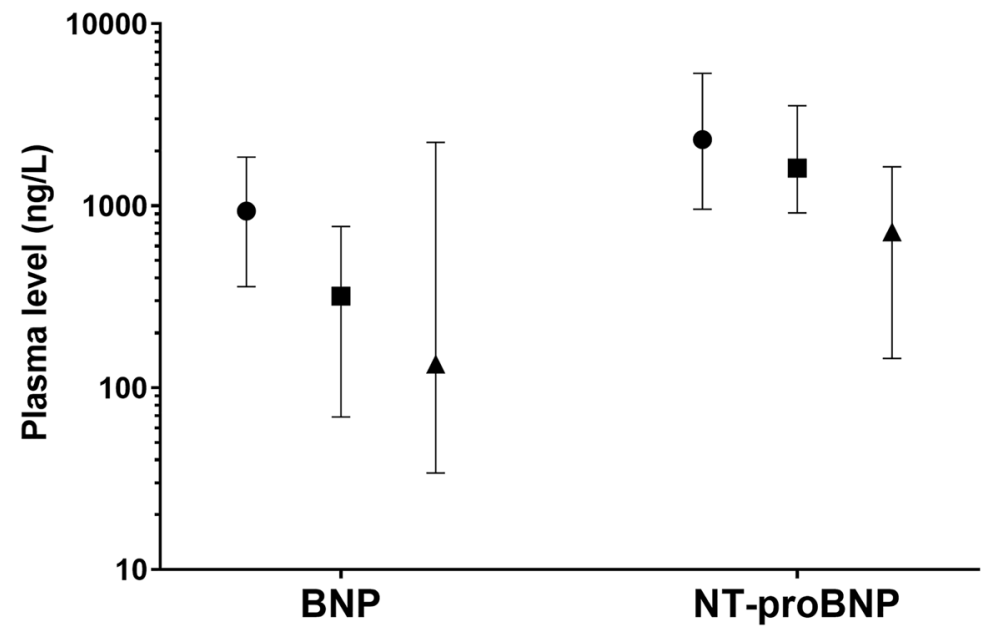

- Baseline

- Month 1

- Month 6

Table 2 Adverse events and treatment-emergent adverse events occurring in the full analysis set

\begin{tabular}{|c|c|c|}
\hline & AEs in the full analy & \\
\hline & No. of patients $(\%)$ & No. of events \\
\hline Any AE & $205(31.3)$ & 359 \\
\hline Any TEAE & $205(31.3)$ & 359 \\
\hline Any serious TEAE & $123(18.8)$ & 199 \\
\hline TEAEs leading to death & $37(5.6)$ & 45 \\
\hline Any drug-related TEAE & $44(6.7)$ & 60 \\
\hline Any serious drug-related TEAE & $11(1.7)$ & 14 \\
\hline Drug-related TEAEs of special interest & $6(0.9)^{\mathrm{a}}$ & 7 \\
\hline Specific events by System Organ Class & & \\
\hline Preferred Term & & \\
\hline Cardiac disorders & $98(15.0)$ & 129 \\
\hline Heart failure & $43(6.6)$ & 48 \\
\hline Atrial fibrillation & $9(1.4)$ & 10 \\
\hline Congestive cardiomyopathy & $7(1.1)$ & 10 \\
\hline Bradycardia & $6(0.9)$ & 6 \\
\hline Infections and infestations & $17(2.5)$ & 20 \\
\hline Pneumonia & $8(1.2)$ & 8 \\
\hline General disorders/administration site conditions & $34(5.2)$ & 36 \\
\hline Death & $16(2.4)$ & 16 \\
\hline Respiratory, thoracic or mediastinal disorders & $27(4.1)$ & 32 \\
\hline Cough & $8(1.2)$ & 8 \\
\hline Upper respiratory tract infection & $8(1.2)$ & 8 \\
\hline Eye disorders & $10(1.5)$ & 13 \\
\hline Photopsia & $6(0.9)$ & 6 \\
\hline
\end{tabular}

$A E$ adverse event, TEAE treatment-emergent adverse event

${ }^{a}$ Incidence $0.9 \%$ (95\% confidence interval $0.337-1.983$ )

proportional to HR [1]; thus, reducing HR in patients with HF should increase myocardial contractility, and this has been demonstrated with ivabradine [2]. In addition, the ivabradine-induced HR reduction increases the diastolic interval, which in turn, improves coronary perfusion [28] and results in greater ventricular filling during diastole, which increases stroke volume [29]. Together, these mechanisms reduce myocardial oxygen demand and explain improvements in cardiac output associated with ivabradine treatment [29]. These mechanisms are achieved without 
negative inotropic effects or a negative impact on conduction [30, 31]. Long-term, ivabradine treatment can delay or even reverse myocardial apoptosis and remodeling induced by energy deficiency [14], ultimately improving cardiac function and QoL.

Until now, the main source of clinical support for the use of ivabradine in Chinese patients with HF has been a subanalysis of 225 patients in the SHIFT trial [20]. As described earlier, the mean placebo-corrected reduction in $\mathrm{HR}$ in patients receiving ivabradine was $12.1 \mathrm{bpm}$ at 28 days. However, the primary endpoint of the SHIFT trial was a composite of cardiovascular death or hospitalization for worsening HF; in the Chinese subpopulation, ivabradine was associated with a $44 \%$ reduction in the incidence of this outcome versus placebo (hazard ratio [HR] 0.56, 95\% CI 0.33-0.97; $p=0.039$ ), after a mean follow-up of 15.6 months. Because of the study design and type of patients included in the POSITIVE study, we did not investigate the effects of ivabradine on mortality or hospitalization rates.

The mean age of patients in our cohort (54 years) is younger than previously reported in Chinese registry studies of hospitalized patients with HF [32, 33]. For example, the mean age of patients hospitalized with HFrEF in the HERO registry was 66.6 years [32]. This likely reflects the underlying cause of $\mathrm{HF}$ in our population, since $45.6 \%$ had dilated cardiomyopathy and $36.2 \%$ had coronary artery disease, and patients with dilated cardiomyopathy tend to be 5-10 years younger than patients with HF caused by other etiologies [34]. In contrast, coronary artery disease was the leading cause of HF in the China PEACE study (affecting $60.6 \%$ of patients), in which the median age was 73 years [33]. In addition, approximately $25 \%$ of patients in our cohort were outpatients, whereas the previous registry studies were in hospitalized patients, which may also explain the difference in age between our cohort and previous reports. The mean age of our cohort may also reflect the type of patients who are prescribed ivabradine, since data show that HF patients who would meet the criteria for entry to the SHIFT study are significantly younger than patients who would not have been candidates for ivabradine in that study [35].

This study has limitations inherent in the nature of its design (open-label, observational), which may have resulted in bias towards overestimating the treatment effect. Other limitations are the absence of a placebo group and the fact that changes in background therapies might also lead to overestimation of the treatment effect. In addition, the results of observational studies can be undermined by missing data, because (in contrast to clinical trials) measurements of interest are not routinely or regularly performed in clinical practice. Lastly, our findings should be regarded as preliminary; the POSITIVE study is ongoing, with more than 1000 patients enrolled to date and a final analysis to be performed once enrolment and follow-up are complete.

\section{Conclusion}

The POSITIVE study is a multicenter, prospective, observational study of ivabradine in the treatment of Chinese patients with HF and is the largest study of ivabradine undertaken to date in a Chinese population. Interim data show that ivabradine treatment reduced HR over 6 months and was associated with statistically significant improvements in NYHA class and clinically meaningful changes in QoL scores. These benefits were accompanied by good general tolerability.

\section{Appendix}

POSITIVE Study Centers (Principal Investigators): Zhongshan Hospital, Fudan University, Shanghai (Dr Junbo Ge); The First People's Hospital of Shunde, Foshan (Dr Jianhua Lu); Qingdao Fuwai Cardiovascular Hospital, Qingdao (Dr Xianyan Jiang); Huizhou Municipal Central Hospital, Huizhou (Dr Yuansheng Shen); Tongji Hospital, Tongji Medical College of HUST, Wuhan (Dr Xiaomei Guo); Jinan Central Hospital, Jinan (Dr Guohai $\mathrm{Su}$ ); Peking Union Medical College Hospital, Beijing (Dr Shuyang Zhang); Beijing Hospital, Beijing (Dr Jiefu Yang); The First Affiliated Hospital of Zhengzhou University, Zhengzhou (Dr Luosha Zhao); Fuwai Hospital Chinese Academy of Medical Sciences, Shenzhen (Dr Yan Xu); Fuwai Hospital, Chinese Academy of Medical Sciences \& Peking Union Medical College, Beijing (Dr Yuhui Zhang); The Fourth People's Hospital of Chongqing, Chongqing (Dr Jun Xiao); Xiamen Cardiovascular Hospital Xiamen University, Xiamen (Dr Wenhui Liu); Tongren Hospital Shanghai Jiao Tong University School of Medicine, Shanghai (Dr Zhaohui Qiu); Nanhai Hospital of Southern Medical University, Foshan (Dr Jingwen Huang); Qilu Hospital of Shandong University (Qingdao), Qingdao (Dr Zhiming Ge); Zhongshan People's Hospital, Zhongshan (Dr Li Feng); Yanan University Affiliated Hospital, Yan'an (Dr Feng Gao); Tongji Hospital of Tongji University, Shanghai (Dr Haoming Song); Dalian Municipal Central Hospital, Dalian (Dr Xiaoqun Zheng); The Second People's Hospital of Hunan Province (Brain Hospital of Hunan Province), Changsha (Dr Xiaoli Chai); Beijing Tsinghua Changgung Hospital, Beijing (Dr Ping Zhang); The First Affiliated Hospital of Xinjiang Medical University, Urumchi (Dr Baopeng Tang); The Second Affiliated Hospital of Nanchang University, Nanchang (Dr 
Xiaoshu Cheng); Puyang Oilfield General Hospital, Puyang (Dr Hengliang Wang); Kunming Municipal Hospital of Traditional Chinese Medicine, Kunming (Dr Yanrong Jin); Longgang District Central Hospital of Shenzhen, Shenzhen (Dr Shengxian Fang); Peking University First Hospital, Beijing (Dr Wenhui Ding); Heilongjiang Provincial Hospital, Harbin (Dr Zheng Wang); Hebei General Hospital, Shijiazhuang (Dr Xiaoyong Qi); Henan Provincial Chest Hospital, Zhengzhou (Dr Kejun Huang); Shanghai Tenth People's Hospital, Shanghai (Dr Yawei $\mathrm{Xu}$ ); Emergency General Hospital, Beijing (Dr Di Wu); Zhuji City People's Hospital, Zhu Ji (Dr Caizhen Qian); Tianjin Medical University General Hospital, Tianjin (Dr Yuemin Sun); Shanxi Cardiovascular Hospital, Taiyuan (Dr Huiyuan Han); The Affiliated Hospital of Qingdao University, Qingdao (Dr Shanglang Cai); Shanghai East Hospital, Shanghai (Dr Ying Li); The First Affiliated Hospital, Sun Yat-Sen University, Guangzhou (Dr Yugang Dong); The Third Affiliated Hospital, Sun Yat-Sen University, Guangzhou (Dr Jieming Zhu); Huaihe Hospital of Henan University, Kaifeng (Dr Ruili He); The Second Affiliated Hospital of Guiyang University of Chinese Medicine, Guiyang (Dr Tao Xu); Hunan Provincial People's Hospital, Changsha (Dr Zhaofen Zheng); Jiangsu Province Hospital, Nanjing (Dr Xiangqing Kong); The Third People's Hospital of Hubei Province, Wuhan (Dr Wenxia Zong); Xi'an No.3 Hospital, Xi'an (Dr Kang Cheng); Fujian Provincial Hospital, Fuzhou (Dr Dingshan Huang); Civil Aviation General Hospital, Beijing (Dr Zeyuan Fan); Sir Run Run Shaw Hospital, School of Medicine, Zhejiang University, Hangzhou (Dr Guosheng Fu); Peking University Third Hospital, Beijing (Dr Zhaoping Li); Beijing Anzhen Hospital, Capital Medical University, Beijing (Dr Xiaohui Liu); The First People's Hospital of Yunnan Province, Kunming (Dr Hong Zhang); Affiliated Hospital of Zunyi Medical University, Zunyi (Dr Bei Shi); The Third Affiliated Hospital of Southern Medical University, Guangzhou (Dr Tao Zhou); Ankang Central Hospital, Ankang (Dr Guidong Shen); Ganzhou People's Hospital, Ganzhou (Dr Jun Luo); First Affiliated Hospital of Gannan Medical University, Ganzhou (Dr Yiming Zhong); West China Hospital, Sichuan University, Chengdu (Dr Qing Zhang); Huazhong University of Science and Technology Union Shenzhen Hospital (Nanshan Hospital), Shenzhen (Dr Peiyi Xie); Peking University International Hospital, Beijing (Dr Xiaohui Liu); The Second Hospital of Heibei Medical University, Shijiazhuang (Dr Fan Liu); Zhejiang Provincial Hospital of Chinese Medicine, Hangzhou (Dr Yuangang Qiu); Xi'an No.1 Hospital, Xi-an (Dr Manli Cheng).

Acknowledgements The authors would like to thank the individuals who participated in this study, and ClinChoice Medical (TIANJIN) Co.,
Ltd for assistance with the statistical analyses. They would also like to thank Richard Crampton of Springer Healthcare Communications, who edited this manuscript. This editorial assistance was funded by Servier, France.

\section{Declarations}

Funding This study was sponsored by Servier (Tianjin) Pharmaceutical Co., Ltd. The Article Processing Charge was funded by Servier, France.

Conflicts of interest/Competing Interests Zhou Jingmin, Xu Yamei, Zhang Yuhui, Ding Wenhui, Tang Baopeng, Qian Caizhen, Han Huiyuan, and Ge Junbo have received fees, research grants, or both, from Servier.

Ethics approval/Consent to participate Informed consent was obtained from all study participants. This study was approved by the Ethics Committee of Zhongshan Hospital Fudan University (ref: B2016-118). The study was registered at the ISRCTN registry (registration number: 11703380).

Consent for publication Not applicable.

Availability of data and material The datasets generated and/or analyzed during the current study are available from the corresponding author on reasonable request.

Code availability Not applicable.

Author contributions All authors participated in the design of the study, interpretation of the data, and writing of the article. The statistical analysis was undertaken independently by ClinChoice Medical (TIANJIN) Co., Ltd. All named authors meet the International Committee of Medical Journal Editors (ICMJE) criteria for authorship for this article, take responsibility for the integrity of the work as a whole, and have read and approved this version to be published.

Open Access This article is licensed under a Creative Commons Attribution-NonCommercial 4.0 International License, which permits any non-commercial use, sharing, adaptation, distribution and reproduction in any medium or format, as long as you give appropriate credit to the original author(s) and the source, provide a link to the Creative Commons licence, and indicate if changes were made. The images or other third party material in this article are included in the article's Creative Commons licence, unless indicated otherwise in a credit line to the material. If material is not included in the article's Creative Commons licence and your intended use is not permitted by statutory regulation or exceeds the permitted use, you will need to obtain permission directly from the copyright holder. To view a copy of this licence, visit http://creativecommons.org/licenses/by-nc/4.0/.

\section{References}

1. Bohm M, La Rosee K, Schmidt U, Schulz C, Schwinger RH, Erdmann E. Force-frequency relationship and inotropic stimulation in the nonfailing and failing human myocardium: implications for the medical treatment of heart failure. Clin Investig. 1992;70:421-5. https://doi.org/10.1007/BF00235525.

2. Reil JC, Tardif JC, Ford I, Lloyd SM, O'Meara E, Komajda $\mathrm{M}$, et al. Selective heart rate reduction with ivabradine unloads 
the left ventricle in heart failure patients. J Am Coll Cardiol. 2013;62:1977-85. https://doi.org/10.1016/j.jacc.2013.07.027.

3. Bache RJ, Cobb FR. Effect of maximal coronary vasodilation on transmural myocardial perfusion during tachycardia in the awake dog. Circ Res. 1977;41:648-53. https://doi.org/10.1161/01.res. 41.5.648.

4. Laurent D, Bolene-Williams C, Williams FL, Katz LN. Effects of heart rate on coronary flow and cardiac oxygen consumption. Am J Physiol. 1956;185:355-64. https://doi.org/10.1152/ajple gacy.1956.185.2.355.

5. Heusch G. Heart rate and heart failure. Not a simple relationship. Circ J. 2011;75:229-36. https://doi.org/10.1253/circj.cj-10-0925.

6. Laskey WK, Alomari I, Cox M, Schulte PJ, Zhao X, Hernandez $\mathrm{AF}$, et al. Heart rate at hospital discharge in patients with heart failure is associated with mortality and rehospitalization. J Am Heart Assoc. 2015. https://doi.org/10.1161/jaha.114.001626.

7. Logeart D, Seronde MF. Raised heart rate at discharge after acute heart failure is an independent predictor of one-year mortality. Eur Heart J. 2012;33:485.

8. Böhm M, Swedberg K, Komajda M, Borer JS, Ford I, DubostBrama A, et al. Heart rate as a risk factor in chronic heart failure (SHIFT): the association between heart rate and outcomes in a randomised placebo-controlled trial. Lancet. 2010;376:886-94. https://doi.org/10.1016/s0140-6736(10)61259-7.

9. Ponikowski P, Voors AA, Anker SD, Bueno H, Cleland JG, Coats AJ, et al. 2016 ESC Guidelines for the diagnosis and treatment of acute and chronic heart failure: The Task Force for the diagnosis and treatment of acute and chronic heart failure of the European Society of Cardiology (ESC). Developed with the special contribution of the Heart Failure Association (HFA) of the ESC. Eur J Heart Fail. 2016;18:891-975. https://doi.org/10.1002/ejhf.592.

10. Yancy CW, Jessup M, Bozkurt B, Butler J, Casey DE Jr, Colvin MM, et al. 2017 ACC/AHA/HFSA focused update of the 2013 $\mathrm{ACCF} / \mathrm{AHA}$ guideline for the management of heart failure: a report of the American College of Cardiology/American Heart Association Task Force on clinical practice guidelines and the Heart Failure Society of America. J Am Coll Cardiol. 2017;70:776-803. https://doi.org/10.1016/j.jacc.2017.04.025.

11. Yancy CW, Jessup M, Bozkurt B, Butler J, Casey DE Jr, Colvin MM, et al. 2016 ACC/AHA/HFSA focused update on new pharmacological therapy for heart failure: an update of the 2013 ACCF/AHA guideline for the management of heart failure: a report of the American College of Cardiology/American Heart Association Task Force on clinical practice guidelines and the Heart Failure Society of America. J Am Coll Cardiol. 2016;68:1476-88. https://doi.org/10.1016/j.jacc.2016.05.011.

12. Yancy CW, Jessup M, Bozkurt B, Butler J, Casey DE Jr, Drazner $\mathrm{MH}$, et al. $2013 \mathrm{ACCF} / \mathrm{AHA}$ guideline for the management of heart failure: a report of the American College of Cardiology Foundation/American Heart Association Task Force on practice guidelines. Circulation. 2013;128:e240-327. https://doi.org/10. 1161/CIR.0b013e31829e8776.

13. Swedberg K, Komajda M, Böhm M, Borer JS, Ford I, DubostBrama A, et al. Ivabradine and outcomes in chronic heart failure (SHIFT): a randomised placebo-controlled study. Lancet. 2010;376:875-85. https://doi.org/10.1016/s0140-6736(10) 61198-1.

14. Tardif JC, O’Meara E, Komajda M, Bohm M, Borer JS, Ford I, et al. Effects of selective heart rate reduction with ivabradine on left ventricular remodelling and function: results from the SHIFT echocardiography substudy. Eur Heart J. 2011;32:2507-15. https://doi.org/10.1093/eurheartj/ehr311.

15. Zugck C, Martinka P, Stöckl G. Ivabradine treatment in a chronic heart failure patient cohort: symptom reduction and improvement in quality of life in clinical practice. Adv Ther. 2014;31:961-74. https://doi.org/10.1007/s12325-014-0147-3.
16. Zugck C, Störk S, Stöckl G. Long-term treatment with ivabradine over 12 months in patients with chronic heart failure in clinical practice: effect on symptoms, quality of life and hospitalizations. Int J Cardiol. 2017;240:258-64. https://doi.org/10.1016/j.ijcard. 2017.03.131.

17. Zachariah D, Stevens D, Sidorowicz G, Spooner C, Rowell N, Taylor J, et al. Quality of life improvement in older patients with heart failure initiated on ivabradine: results from the UK multi-centre LIVE:LIFE prospective cohort study. Int J Cardiol. 2017;249:313-8. https://doi.org/10.1016/j.ijcard.2017.08.001.

18. Green CP, Porter CB, Bresnahan DR, Spertus JA. Development and evaluation of the Kansas City Cardiomyopathy Questionnaire: a new health status measure for heart failure. J Am Coll Cardiol. 2000;35:1245-55. https://doi.org/10.1016/s0735-1097(00) 00531-3.

19. Spertus JA, Jones PG, Sandhu AT, Arnold SV. Interpreting the Kansas City Cardiomyopathy Questionnaire in clinical trials and clinical care: JACC state-of-the-art review. J Am Coll Cardiol. 2020;76:2379-90. https://doi.org/10.1016/j.jacc.2020.09.542.

20. Hu DY, Huang DJ, Yuan ZY, Zhao RP, Yan XW, Wang MH. Efficacy and safety analysis of ivabradine hydrochloride treatment of Chinese patients with chronic heart failure: subgroup analysis of Chinese patients in the SHIFT study [in Chinese]. Zhonghua Xin Xue Guan Bing Za Zhi. 2017;45:190-7. https:// doi.org/10.3760/cma.j.issn.0253-3758.2017.03.005.

21. Lopatin YM, Cowie MR, Grebennikova AA, Sisakian HS, Pagava ZM, Hayrapetyan HG, et al. Optimization of heart rate lowering therapy in hospitalized patients with heart failure: insights from the Optimize Heart Failure Care Program. Int J Cardiol. 2018;260:113-7. https://doi.org/10.1016/j.ijcard.2017. 12.093.

22. Chinese guidelines for the diagnosis and treatment of heart failure 2018 [in Chinese]. Zhonghua Xin Xue Guan Bing Za Zhi. 2018;46:760-89. https://doi.org/10.3760/cma.j.issn.0253-3758. 2018.10.004

23. Moser DK, Yamokoski L, Sun JL, Conway GA, Hartman KA, Graziano JA, et al. Improvement in health-related quality of life after hospitalization predicts event-free survival in patients with advanced heart failure. J Card Fail. 2009;15:763-9. https://doi. org/10.1016/j.cardfail.2009.05.003.

24. Ekman I, Cleland JG, Swedberg K, Charlesworth A, Metra M, Poole-Wilson PA. Symptoms in patients with heart failure are prognostic predictors: insights from COMET. J Card Fail. 2005;11:288-92. https://doi.org/10.1016/j.cardfail.2005.03.007.

25. Alpert CM, Smith MA, Hummel SL, Hummel EK. Symptom burden in heart failure: assessment, impact on outcomes, and management. Heart Fail Rev. 2017;22:25-39. https://doi.org/ 10.1007/s10741-016-9581-4.

26. Ekman I, Chassany O, Komajda M, Bohm M, Borer JS, Ford I, et al. Heart rate reduction with ivabradine and health related quality of life in patients with chronic heart failure: results from the SHIFT study. Eur Heart J. 2011;32:2395-404. https://doi. org/10.1093/eurheartj/ehr343.

27. Koroma TR, Samura SK, Cheng Y, Tang M. Effect of ivabradine on left ventricular diastolic function, exercise tolerance and quality of life in patients with heart failure: a systemic review and meta-analysis of randomized controlled trials. Cardiol Res. 2020;11:40-9. https://doi.org/10.14740/cr958.

28. Colin P, Ghaleh B, Monnet X, Su J, Hittinger L, Giudicelli JF, et al. Contributions of heart rate and contractility to myocardial oxygen balance during exercise. Am J Physiol Heart Circ Physiol. 2003;284:H676-82. https://doi.org/10.1152/ajpheart. 00564.2002.

29. De Ferrari GM, Mazzuero A, Agnesina L, Bertoletti A, Lettino M, Campana $\mathrm{C}$, et al. Favourable effects of heart rate reduction with intravenous administration of ivabradine in patients with advanced 
heart failure. Eur J Heart Fail. 2008;10:550-5. https://doi.org/10. 1016/j.ejheart.2008.04.005.

30. Camm AJ, Lau CP. Electrophysiological effects of a single intravenous administration of ivabradine (S 16257) in adult patients with normal baseline electrophysiology. Drugs R D. 2003;4:83-9. https://doi.org/10.2165/00126839-200304020-00001.

31. Manz M, Reuter M, Lauck G, Omran H, Jung W. A single intravenous dose of ivabradine, a novel I(f) inhibitor, lowers heart rate but does not depress left ventricular function in patients with left ventricular dysfunction. Cardiology. 2003;100:149-55. https://doi. org/10.1159/000073933.

32. Li L, Liu R, Jiang C, Du X, Huffman MD, Lam CSP, et al. Assessing the evidence-practice gap for heart failure in China: the Heart Failure Registry of Patient Outcomes (HERO) study design and baseline characteristics. Eur J Heart Fail. 2020;22:646-60. https:// doi.org/10.1002/ejhf.1630.
33. Yu Y, Gupta A, Wu C, Masoudi FA, Du X, Zhang J, et al. Characteristics, management, and outcomes of patients hospitalized for heart failure in China: the China PEACE Retrospective Heart Failure Study. J Am Heart Assoc. 2019;8: e012884. https://doi. org/10.1161/JAHA.119.012884.

34. Seferovic PM, Polovina MM, Coats AJS. Heart failure in dilated non-ischaemic cardiomyopathy. Eur Heart J Suppl. 2019;21:M40 3. https://doi.org/10.1093/eurheartj/suz212.

35. Das D, Savarese G, Dahlstrom U, Fu M, Howlett J, Ezekowitz JA, et al. Ivabradine in heart failure: the representativeness of SHIFT (Systolic Heart Failure Treatment With the IF Inhibitor Ivabradine Trial) in a broad population of patients with chronic heart failure. Circ Heart Fail. 2017;10:e004112. https://doi.org/10.1161/CIRCH EARTFAILURE.117.004112. 